\title{
Manuka Honey: A Tissue Engineering Essential Ingredient
}

\section{Shannon L Speer, Gretchen E Schreyack and Gary L Bowlin*}

Department of Biomedical Engineering, The University of Memphis, Memphis, TN USA

Keywords: Manuka honey; Tissue engineering; Templates; Tissue regeneration

\section{Introduction}

A renewed interest in medical-grade honey is revitalizing the clinical and biomedical engineering communities. The primary driving force for this revitalization has been the goal of developing non-antibiotic alternatives and effective new ways to prevent and fight bacterial infections. This application of honey is not unexpected or new since the use of medicinal honey simply fell out of fashion when antibiotics revolutionized medicine over one hundred years ago. Prior to antibiotics, and dating back as far as 600 B.C., honey had been extensively used in the treatment of infections and burns [1]. More importantly, the broad antimicrobial spectrum of honey makes it a great and powerful alternative to antibiotics when dealing with wound infections. However, care must be used in selection because the antimicrobial spectrum of the honey is very much dependent on the type (source of nectar) of honey used [2]. For this reason, a specific honey, Manuka honey, appears to be leading the charge in transforming the medical industry due to its unique composition and derived functionality. Its functionality includes a broadband anti-bacterial, anti-inflammatory, and enhanced wound healing capacity that may be the tissue engineering trifecta and deserves further consideration as an essential tissue engineering ingredient (i.e. incorporation into templates).

Manuka honey is typically monofloral and derived from the nectar of a flowering scrub-tree native to New Zealand and southeast Australia called Leptospermum scoparium. The unique manuka factor (UMF) in Manuka honey is the methylglyoxal (MGO) level which is responsible for its anti-bacterial effect. Additionally, this non-peroxide property of Manuka honey is important because it will not cause damage to adjacent healthy tissue in sharp contrast to general honey that uses hydrogen peroxide as its active component to kill bacteria. Research has also shown that the low $\mathrm{pH}$ of 3.2-4.5 is a powerful property of honey that kills bacteria with an added benefit of also potentially contributing to the induction of angiogenesis [4,5]. Furthermore, the ability of honey to inactivate free iron is extremely efficient at killing bacteria [6] More importantly for tissue engineering applications, Manuka honey causes increased healing and a reduction in the overall healing time in wound sites. This is directly related to the inflammatory response as Manuka honey enhances the suppression of inflammatory cells and the proliferation of epithelial and fibroblasts $[6,7]$. Thus, leading to one looming question - why is Manuka honey currently used so rarely clinically, knowing the fact that honey has been used for thousands of years as such a safe and effective treatment for infected wounds and burns? As tissue engineers, could this be a missing essential ingredient in tissue engineering template design, especially for acellular tissue regenerative templates designed to promote in situ regeneration?

\section{Methylgloxal: A Unique Contributing Factor}

As noted, the MGO in Manuka honey is responsible for antibacterial activity and creates an environment in which bacteria cannot build a resistance to honey [3]. In particular, Manuka honey will likely continue to be favored when dealing with wound dressings because of its noted non-peroxide activity. Most other varieties of honey rely on peroxide activity to kill bacteria, but this peroxide activity can damage surrounding tissues and decomposes when exposed to heat, the enzyme catalase breaking down hydrogen peroxide in the body, thus rendering most general honey varieties rather ineffective for use in wound repair [3]. As it relates to the MGO content, it has been observed that there is a correlation between the MGO content and the effectiveness of the honey's ability to clear bacteria from infected wounds [3]. Therefore, it can be further suggested that the Manuka honey with a higher MGO content should be utilized based on availability and cost (currently the price significantly increases with MGO content due to limited availability). Manuka honey has a significant therapeutic benefit when the UMF equivalent level is between $12+-19+$ (on a scale of $2+$ to $30+$, with values above $20+$ considered extremely high anti-bacterial benefit but also very expensive and not easily obtained) [8].

One of the more current and prevalent clinical challenges is Methicillin-resistant Staphylococcus aureus (MRSA) which is a common bacterium that causes problems with wound infections after surgery. Notably, a study has shown evidence of healing and eradication of MRSA in patients who were treated with Manuka honey when anitibotics alone could not clear the infection [9]. Another study also provided evidence that the functionality of medical honey was necessary in eliminating MRSA in patients that had infections which persisted for four months despite the continued use of antibiotics [9]. In summary, Manuka honey has demonstrated the ability to stop bacterial (MRSA and E. Coli) growth by in vitro studies and continues to show promise in clinical trials for enhanced wound healing while continuing to be an anti-bacterial and anti-inflammatory agent [10].

\section{Manuka Honey as it Currently Relates to Tissue Engineering}

In the field of tissue engineering, a central area of focus has been on engineering biomaterial-based templates aimed at facilitating and guiding new tissue formation by a variety of approaches. A common theme in designing these tissue regeneration templates remains the necessity for biocompatibility and how does this biocompatibility determine what biomaterial(s) and overall form/architecture can be used for facilitating and guiding regeneration [11]. Historically, and much too often, a tissue regeneration template's biocompatibility has been based upon simple "inertness" upon physiological interaction [11]. As recently presented by Dr. Williams and in order to obtain a template

*Corresponding author: Gary L Bowlin, Professor \& Herbert Herff Chair of Excellence, Director, Tissue Template Engineering and Regeneration Laboratory, Department of Biomedical Engineering, The University of Memphis, Memphis, TN 38152,USA, Tel: 901-678-2670; E-mail: glbowlin@memphis.edu

Received August 01, 2015; Accepted August 03, 2015; Published August 10, 2015

Citation: Speer SL, Schreyack GE, Bowlin GL (2015) Manuka Honey: A Tissue Engineering Essential Ingredient. J Tissue Sci Eng 6: e130. doi:10.4172/21577552.1000e130

Copyright: @ 2015 Speer SL, et al. This is an open-access article distributed under the terms of the Creative Commons Attribution License, which permits unrestricted use, distribution, and reproduction in any medium, provided the original author and source are credited. 
that truly facilitates and guides the desired regeneration, the ideal template characteristics should be more complex and include: capability of replicating the microenvironment of tissue-specific cells, adaptability to the microenvironment, mimicking tissue mechanical properties, optimized surface energy, capacity for reciprocal communication via direct molecular signaling of interacting cells, provide appropriate shape and size of the desired tissue, optimized for each specific tissue development, and composed of material that is non-cytotoxic [11]. Taking the criteria into consideration, how can we design templates that facilitate and guide complex tissue regeneration without the associated bacterial and inflammation-driven complications seen historically?

While the clinical use of Manuka honey has been highly focused on wound dressings, the areas of application should now quickly begin to broaden and include Manuka honey incorporation into tissue regeneration templates for the following reasons: the ability to prevent infections, anti-inflammatory capacity, and capacity to enhance healing through a variety of potential mechanisms. A study published by our group examined the response of macrophages, fibroblasts, and endothelial cells to cell culture media supplemented with lyophilized preparation rich in growth factors (PRGF) derived from platelet-rich plasma (PRP) and Manuka honey [12]. This study demonstrated that Manuka honey may have a potential additive effect over just the popular PRP-based therapies and its use may potentially enhance or accelerate regeneration, further detailed in vivo evaluations necessary. Additionally, another study showed that poly (vinyl alcohol) (PVA) and Manuka honey could be incorporated into a composite dressing [13]. PVA has excellent biocompatibility and is easily formed into templates (i.e. good fiber forming qualities), which allows the incorporation of Manuka honey for enhanced regeneration (controlled release delivery vehicle) versus a simple polymeric-based template while at the same time minimizing opportunities of bacterial colonization. The controlled release delivery vehicle is another aspect of particular note because current honey therapies are often deemed ineffective for one primary reason, at body temperature the honey becomes more fluid and disperses rapidly from the site of application. Another potential mechanism of Manuka honey is its capacity to stimulate cytokines that promote tissue growth through the proliferation of fibroblasts and epithelial cells and leads to a strong presence of macrophages at the wound site when a wound is dressed with Manuka honey [6]. Moreover, in burn wounds, honey has been seen to promote re-epithelialization [6]. Thus, evidence of a potential tissue regeneration trifecta and that further evaluations of templates incorporating Manuka honey is warranted.

\section{Potential Concerns with Manuka Honey}

Although there has been considerable effort and research regarding the antimicrobial and topical healing effects of Manuka honey, there has not been a strong focus on the possible adverse effects of Manuka honey on wound healing or upon use internally. As a precaution, research has shown that honey dressings should not be applied to patients who have an allergy to bee stings [6]. Furthermore, the low $\mathrm{pH}$ and high acidity of Manuka honey can cause temporary pain, stinging, that dissipates quickly in patients when there is high inflammation at the wound site [6]. One of the complicating factors, as noted previously, is honey's fluidity upon application, which makes it difficult to determine the efficacy of Manuka honey with a complex patient population [6]. Another concern is the risk of introducing microorganism contained in the honey upon template incorporation, a scenario easily mitigated by proper sterilization. Yet another concern or risk is that of contaminating the wound with pollen that may not have been cleaned from filtering causing an allergic reaction [6].
As noted, the critical factor that renders Manuka honey its unique functionality is MGO. This beneficial factor can be detrimental for the healing of diabetic ulcers. Research has shown that MGO and advanced glycation end products can actually prevent wound healing when the patient is a diabetic by enhancing the activity of neutrophils causing chronic inflammation and reduced healing [2].

In a single study examining the effects of internally applied Manuka honey, the ototoxic effects have been demonstrated in an animal model. The purpose of this study was to examine ototoxicity in the chinchilla model with a $50 \%$ concentration of Manuka honey (12+ UMF equivalent) [14]. This concentration is well above the 4-5\% Manuka honey (16+ UMF equivalent) solution shown effective against MRSA and P. aeruginosa [15]. A myringotomy was performed on the chinchillas which required an incision to relieve pressure in the middle ear [14]. This is one of the first in vivo trials of Manuka honey exposure internally. The results demonstrated clearly that the animals with exposure to high concentration Manuka honey in the middle ears internally to fight biofilms displayed intense or severe inflammatory changes which resulted in facial paralysis, vestibuleotoxicity, and hearing loss [14]. Thus, as the potential use of Manuka honey is explored in tissue regeneration templates, the quantity available at the surface and/or release kinetics will be of concern as to not cause local complications and maintain the potential beneficial effects of its use.

\section{Conclusion and Perspective}

While many medical professionals still view the use of Manuka honey, especially as a substitute for antibiotics, with skepticism its potential especially in tissue engineering applications cannot be denied. Manuka honey epitomizes the tissue engineering trifecta, it is anti-bacterial, immunomodulatory, and it enhances wound healing and tissue regeneration. The Manuka honey trifecta may now lead it to being an essential and all-natural ingredient that paves the way for the development of ideal tissue regeneration templates for a variety of applications. Much work is still required regarding the mechanisms in play with a variety of cellular components upon interacting with Manuka honey and the potential enhancement of tissue regeneration. More importantly, it appears that the drawbacks of Manuka honey are limited but very manageable when considering its many potential benefits and applications. Finally, it is clear that Manuka honey dressings are effective, relative to conventional wound dressings, at preventing infection as well as promoting tissue regeneration in many applications. It is imperative that research focus now turns to the potential extensive internal use of Manuka honey and particularly its incorporation into tissue regeneration templates.

\section{References}

1. Udwadia TE (2011) Ghee and honey dressing for infected wounds. Indian J Surg 73: 278-283.

2. Mandal MD, Mandal S. (2011) Honey: its medicinal property and antibacterial activity. Asian Pacific Journal Of Tropical Biomedicine 1: 154-160

3. Alvarez-Suarez JM, Gasparrini M, Forbes-Hernandez TY, Mazzoni L, Francesca G (2014) The composition and biological activity of honey: A focus on Manuka honey. Foods 3: 420-432

4. Villianou NG, Gounari P, Skourtis A, Panagos J, Christos K (2014) Honey and its anti-inflammatory, anti-bacterial and anti-oxidant properties. General Medicine 2: 1-5.

5. Roecken, F (2013). Honey in modern wound treatment-the renaissance of an ancient remedy. Journal of Kleintier-Praxis 58: 617-618.

6. Sharp A (2009) Beneficial effects of honey dressings in wound management Nursing Standard 24: 66-74. 
Citation: Speer SL, Schreyack GE, Bowlin GL (2015) Manuka Honey: A Tissue Engineering Essential Ingredient. J Tissue Sci Eng 6: e130. doi:10.4172/2157-7552.1000e130

Page 3 of 3

7. Tomblin V, Ferguson LR, Han DY, Murray P, Ralf S (2014) Potential pathway of anti-inflammatory effect by New Zealand honeys. International Journal of General Medicine 7:149-158.

8. http://www.beelimitless.com/about-honey/bio-activ-nmf/honey/

9. Blaser G, Santos K, Bode U, Vetter H, Simon A (2007) Effect of medical honey on wounds colonised or infected with MRSA. Journal of Wound Care 16: 325328

10. Lusby PE, Coombes A, Wilkinson JM (2002) Honey: A potent agent for wound healing? Journal of Wound Ostomy and Continence Nursing 29: 295-300.

11. Williams DF (2014) The biomaterials conundrum in tissue engineering. Tissue Engineering 20: 1129-1131.
12. Scott SA, Wolfe PS, Spence AJ, Rodriguez IA, Mc Cool JM, et al. (2012) A preliminary study on the potential of Manuka honey and platelet-rich plasma in wound healing. International Journal of Biomaterials 10: 1-14.

13. Bulman SE, Goswami P, Tronci G, Russel SJ, Carr C (2015) Investigation into the potential use of poly(vinyl alcohol)/methylglyoxal fibres as antibacterial wound dressing components. Journal of Biomaterials Applications 29: 11931200.

14. Aron M, Akinpelu V, Dorion D, Daniel S (2012) Otologic safety of Manuka honey. J Otolaryngol Head Neck Surg 41: S21-S30.

15. Kwakman $\mathrm{PH}$, te Velde AA, de Boer L, Vandenbroucke-Grauls CM, Zatt SA (2011) Two major medicinal honeys have different mechanisms of bactericidal activity. PLoS ONE 6: e17709. 